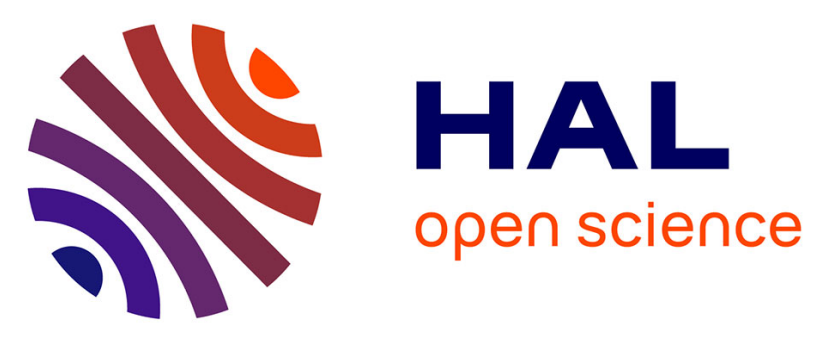

\title{
Intraband and intersubband many-body effects in the nonlinear optical response of single-wall carbon nanotubes
}

Benjamin Langlois, Romain Parret, Fabien Vialla, Yannick Chassagneux, Philippe Roussignol, Carole Diederichs, Guillaume Cassabois, Jean-sébastien Lauret, Christophe Voisin

\section{To cite this version:}

Benjamin Langlois, Romain Parret, Fabien Vialla, Yannick Chassagneux, Philippe Roussignol, et al.. Intraband and intersubband many-body effects in the nonlinear optical response of single-wall carbon nanotubes. Physical Review B: Condensed Matter and Materials Physics (1998-2015), 2015, 92, pp.155423. 10.1103/PhysRevB.92.155423 . hal-01250121

\section{HAL Id: hal-01250121 \\ https://hal.science/hal-01250121}

Submitted on 4 Jan 2016

HAL is a multi-disciplinary open access archive for the deposit and dissemination of scientific research documents, whether they are published or not. The documents may come from teaching and research institutions in France or abroad, or from public or private research centers.
L'archive ouverte pluridisciplinaire HAL, est destinée au dépôt et à la diffusion de documents scientifiques de niveau recherche, publiés ou non, émanant des établissements d'enseignement et de recherche français ou étrangers, des laboratoires publics ou privés. 


\title{
Intraband and intersubband many-body effects in the nonlinear optical response of single-wall carbon nanotubes.
}

\author{
Benjamin Langlois, ${ }^{1}$ Romain Parret, ${ }^{1}$ Fabien Vialla, ${ }^{1}$ Yannick Chassagneux,${ }^{1}$ Philippe Roussignol, ${ }^{1}$ \\ Carole Diederichs, ${ }^{1}$ Guillaume Cassabois, ${ }^{2}$ Jean-Sébastien Lauret, ${ }^{3}$ and Christophe Voisin ${ }^{1, *}$ \\ ${ }^{1}$ Laboratoire Pierre Aigrain, École Normale Supérieure, Université Paris Diderot, \\ UPMC, CNRS UMR8551, 24 rue Lhomond, 75005 Paris, France \\ ${ }^{2}$ Laboratoire Charles Coulomb, Université Montpellier, CNRS UMR5221, 34095 Montpellier, France \\ ${ }^{3}$ Laboratoire Aimé Cotton, ENS Cachan, CNRS, Université Paris Sud, 91405 Orsay, France
}

(Dated: September 22, 2015)

\begin{abstract}
We report on the nonlinear optical response of a mono-chiral sample of $(6,5)$ single-wall carbon nanotubes by means of broad-band two-color pump-probe spectroscopy with selective excitation of the $S_{11}$ excitons. By using a moment analysis of the transient spectra, we show that all the nonlinear features can be accurately accounted for by elementary deformations of the linear absorption spectrum. The photo-generation of $S_{11}$ excitons induces a broadening and a blue shift of both the $S_{11}$ and $S_{22}$ excitonic transitions. In contrast, only the $S_{11}$ transition shows a reduction of oscillator strength, ruling out population up-conversion. These nonlinear signatures result from many-body effects, including phase-space filling, wave-function renormalization and exciton collisions. This framework is sufficient to interpret the magnitude of the observed nonlinearities and stress the importance of intersubband exciton interactions. Remarkably, we show that these intersubband interactions have the same magnitude as the intraband ones and bring the major contribution to the photo-bleaching of the $S_{22}$ excitonic transition upon $S_{11}$ excitation through energy shift and broadening.
\end{abstract}

\section{INTRODUCTION}

Single-wall carbon nanotubes are unique nearly onedimensional nano-structures that trigger a wealth of investigations both regarding academical aspects and applications. The possible geometries of these hollow cylinders, known as chiral species, give rise to a large variety of electronic properties that are uniquely related to their structure $^{1}$. The electronic and optical properties of carbon nanotubes reflect large electronic correlations leading for instance to the formation of strongly bound excitons, even at room temperature ${ }^{2,3}$. At higher excitation densities, electronic correlations give rise to a number of new signatures related to the exciton-exciton interactions, including exciton-exciton annihilation ${ }^{4}$ or the formation of bi-excitons ${ }^{5}$ or trions ${ }^{6}$ below the $S_{11}$ excitonic transition. These nonlinear effects are already successfully exploited for applications as saturable absorbers in ultrafast lasers for instance ${ }^{7}$. In contrast to other semiconducting nano-structures, the magnitude of these interactions is strongly enhanced by the one-dimensional geometry of the nanotubes ${ }^{8}$. Beside excitonic complexes and exciton annihilation, many-body effects also induce broad spectral range optical signatures such as bandgap renormalization or collisional broadening ${ }^{9}$. In contrast to excitonic complexes, such effects are best detectable at photon energies larger than that of the lower excitonic transition.

In most of the previous studies however, such effects were blurred because of the spectral congestion of optical transitions due to the use of poly-chiral suspensions ${ }^{10,11}$. As a consequence, most of the previous pumpprobe studies have been interpreted in terms of simple photo-bleaching $(\mathrm{PB})$ and photo-absorption (PA) bands respectively assigned to state filling effects (Pauli blocking in a molecular picture) and absorption from longlived excited states to upper states ${ }^{11-13}$. This empirical approach to the nonlinear optical properties of carbon nanotubes suffers from two main issues : firstly, the molecular picture does not take properly into account the bosonic nature of the excitonic excitations, and secondly, the PA bands require the introduction of $a d-h o c$ upper states that are not supported by calculations of the electronic structure of the nanotubes.

In this study, we present a broad spectral range investigation of the optical nonlinearities in a mono-chiral ensemble of carbon nanotubes. We focus on the case of a low-energy $\left(S_{11}\right)$ pumping scheme that is more suited to unveil many-body effects beyond simple phase space filling effects. We use an intrinsic analysis method based on the moments of the transient absorption spectra that does not require any assumption about the line-shape of the transitions. We show that the photo-creation of $S_{11}$ excitons induces a broadening and a blue shift of both the $S_{11}$ and $S_{22}$ lines of the order of a few meV. In contrast, the reduction of oscillator strength is observed only for the $S_{11}$ transition, showing that strong changes in the $S_{22}$ absorption band can be obtained through nonlinear intersubband interactions even if the $S_{22}$ population remains negligible. Furthermore, by using combinations of nonlinear signals, we can eliminate the (unknown) exciton density and access in the most possible intrinsic way to the microscopic parameters. All these experimental observations can be accounted for in a simple and unified description of many-body effects in carbon nanotubes, based on a mean field perturbative approach that generalizes to the one-dimensional case the calculations previously developed for two-dimensional nano- 
structures $^{14,15}$.

\section{EXPERIMENTAL SETUP AND SAMPLES}

The sample consists in an aqueous suspension of $(6,5)$ enriched carbon nanotubes encapsulated in micelles purchased from NanoIntegris. The suspension is placed in a 1-mm thick quartz cuvette for the measurements of both the linear and nonlinear properties. The linear absorption $\left(\alpha_{0}\right)$ spectrum is displayed in the inset of Fig. 1. It consists of two main lines at $1.25 \mathrm{eV}$ and $2.2 \mathrm{eV}$ corresponding to the excitonic transitions of the first and second subbands respectively, the so-called $S_{11}$ and $S_{22}$ transitions. In addition to these main features, weaker resonances are attributed to phonon side-bands ${ }^{16}$ and possibly remaining minority chiral species. Finally, these resonances lie on a flat absorption background attributed to both an intrinsic non-resonant contribution ${ }^{17}$ and to various impurities ${ }^{18}$. We delineate those two contributions by means of a thorough comparison of the absorption spectrum with the photo-luminescence excitation spectrum (PLE) of the same suspension, which allows us to access the truly intrinsic absorption of the $(6,5)$ species $^{17}$ in the $[1.4 \mathrm{eV}-3 \mathrm{eV}]$ window.

The nonlinear signals are obtained from co-polarized pump-probe measurements at a sub-picosecond timescale. The pump-probe setup is based on a visible optical parametric amplifier (OPA) pumped at $400 \mathrm{~nm}$ by a frequency doubled amplified Ti:Sapphire laser at a repetition rate of $250 \mathrm{kHz}$. Part of the continuum generated in a sapphire crystal to seed the OPA is picked up to serve as a probe beam, whereas the signal or idler beams of the OPA are used as a pump. The wavelength can be tuned continuously from 445 to $1200 \mathrm{~nm}$ (1 to $2.8 \mathrm{eV}$ ) with a spectral width of the order of $15 \mathrm{meV}$. In the following, the pump energy is fixed at $1.25 \mathrm{eV}$ in resonance with $S_{11}$, except when otherwise specified. The probe beam is further filtered by a monochromator ( $5 \mathrm{~nm}$ bandpass) after passing through the sample ${ }^{19}$. The instrumental response of the setup was measured by stimulated Raman scattering in water and gives an overall temporal resolution of $270 \mathrm{fs}$. The chirp of the probe beam was measured and numerically corrected in the data.

\section{TRANSIENT SPECTRA AND QUANTITATIVE ANALYSIS}

The nonlinear broadband spectrum shown in Fig. 1 consists of photo-absorption $(\Delta \alpha>0)$ and photobleaching $(\Delta \alpha<0)$ bands near each excitonic resonance. The signal in the $S_{22}$ spectral range is about ten times smaller than in the $S_{11}$ range. The shape of the (normalized) nonlinear spectra does not depend on the pumpprobe time-delay (see inset of Fig. 2) nor on the pump wavelength (as already reported in Ref. ${ }^{13}$ for instance). Neither does it depend on the fluence except in the very first picoseconds after excitation at high fluence (above $\left.10^{15} \mathrm{~cm}^{-2}\right)$.

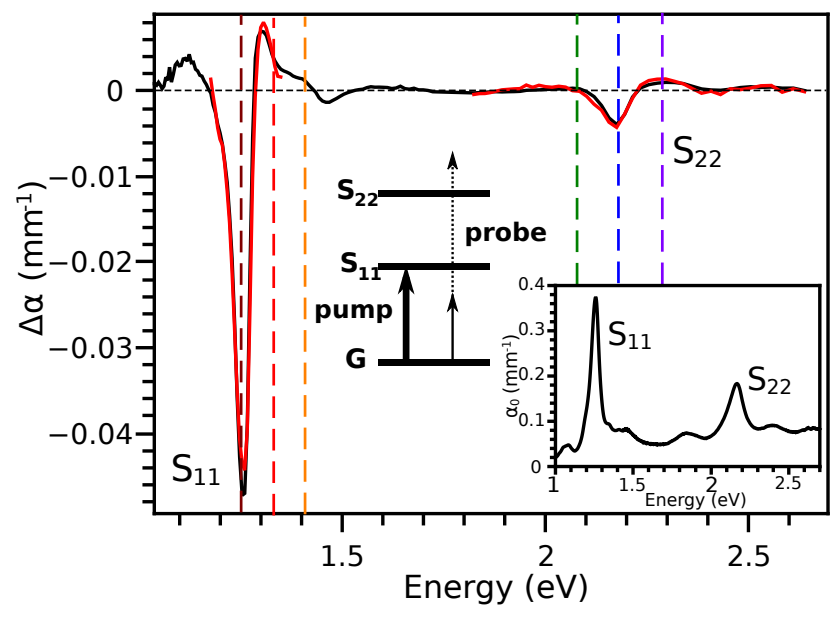

FIG. 1. A broadband nonlinear absorption spectrum (black) 1 ps after $S_{11}$ excitation at a fluence of $2 \times 10^{13}$ photons.cm ${ }^{-2}$. Fit (red) of the data using a direct deformation (including bleaching, shift and broadening) of the linear absorption spectrum. Probe energy references for Fig. 2 (vertical dashed lines). Inset : linear absorption spectrum.

In order to determine the origin of the nonlinear signal, we first focus on the relaxation dynamics of the transient absorption for different probe frequencies (Fig. 2). The relaxation of the transient absorption resonant with $S_{11}$ is known to follow a $\Delta \alpha(t) \propto t^{-1 / 2}$ decay law from few picoseconds up to $1 \mathrm{~ns}$ after excitation of either the $S_{11}$ or $S_{22}$ transitions ${ }^{13,20}$, independently of the pump power ${ }^{21}$. Zhu et al. also showed that the transient absorption at $S_{22}$ follows the same dynamics ${ }^{13}$. This decay is typical of a diffusion limited annihilation reaction between identical particles in a $1 \mathrm{D}$ material $^{22,23}$. In the case of carbon nanotubes, this relaxation is interpreted as excitonexciton annihilation ${ }^{13,21}$ which is known to be significant in strongly confined system ${ }^{24}$. Fig. 2 shows that the same dynamics is also observed in the non-resonant transient absorption on both sides of the excitonic transitions. The $\mathrm{PB}$ and PA bands clearly display the same relaxation dynamics, indicating that all nonlinear features stem from a unique physical origin, namely the dynamics of the lower energy $S_{11}$ excitations.

We now turn to the physical origin of these nonlinear signatures by investigating systematically the spectral shape of the transients. We achieve a simple and consistent description by considering a set of many-body effects, that is able to account for the nonlinear optical signatures on a very wide range of probe energies. These nonlinear signatures are interpreted as consequences of the presence of $S_{11}$ excitons in the nanotube leading to phase space filling, wave-function renormalization and excitonic collisions. These effects reshape all the excitonic resonances and can be accounted for by elementary deformations of each excitonic line $S_{i i}$ : reduction of oscillator strength $\Delta f^{i i}$, energy shift $\Delta E^{i i}$ and broadening 


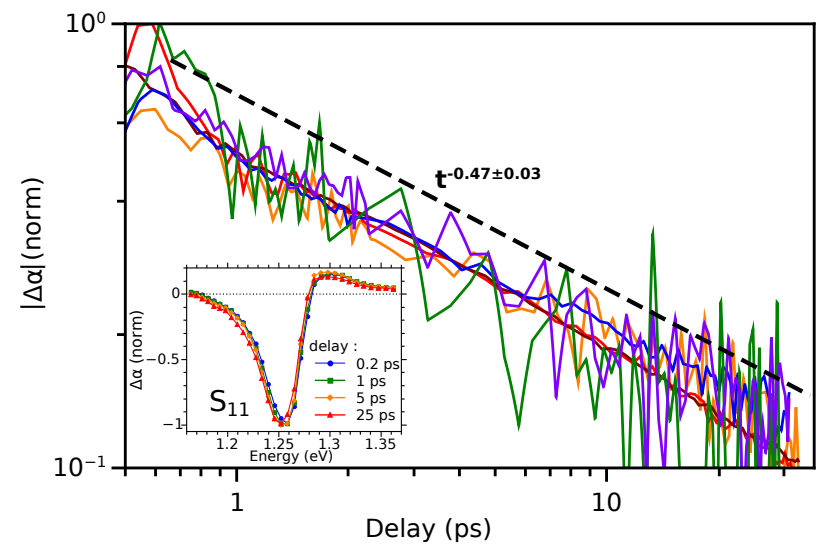

FIG. 2. (color online) Relaxation dynamics of normalized transient absorption after $S_{11}$ excitation for probe energies given by the dashed vertical lines of Fig. 1 (same color code). The black dashed line corresponds to a $t^{-0.47}$ normalized decay. Inset: normalized nonlinear absorption spectra excited at $1.26 \mathrm{eV}$, fluence of $3.2 \times 10^{14}$ photons.cm ${ }^{-2}$, for different pump-probe delays.

$\Delta \Gamma^{i i}$ with respect to the equilibrium values $f_{0}^{i i}, E_{0}^{i i}$ and $\Gamma_{0}^{i i}$.

In a first approach, elementary deformations are separately applied to the linear spectrum near $S_{11}$ and $S_{22}$ resonances in order to reproduce qualitatively the nonlinear spectra. This approach allows to fully reproduce the experimental data as it can be seen in Fig. 1 (red curves). Computing this nonlinear spectrum requires two precautions. Firstly, the spectrum boundaries must be chosen so as to avoid extrinsic contributions such as the ones of minority chiral species, that are not resonantly excited by the pump. Otherwise, the procedure would generate artifacts. Similarly, the influence of multi-excitonic complexes, which is well documented ${ }^{5,25}$, has not been introduced, such complexes giving specific signatures below $1.1 \mathrm{eV}$ in the case of $(6,5)$ nanotubes. Secondly, the extrinsic absorption background has to be subtracted from the linear spectrum before applying any deformation in order to obtain a reliable simulated nonlinear spectrum. This background can be accurately evaluated from PLE measurements ${ }^{17}$. This background correction method is however not applicable near the $S_{11}$ resonance due to overwhelming elastic scattering in the PLE spectrum. Nevertheless, the computed nonlinear spectrum reproduces successfully the PA and PB band profiles, especially their asymmetry and relative amplitudes. This indicates that the three elementary deformations considered in this description are sufficient to reproduce accurately the nonlinear spectra. Reciprocally, we note that all of these three contributions are necessary, except $\Delta f^{22}$. In fact, considering only a reduction of oscillator strength $(\Delta f<0)$ would produce a symmetric $\mathrm{PB}$ band, whereas a simple energy shift $\Delta E$ would give rise to an derivative-like profile composed of a pair $\mathrm{PB}$ and PA bands of similar amplitude, and a plain broad- ening $\Delta \Gamma$ would lead to a PB band surrounded by two symmetrical PA bands.

In order to get a more quantitative insight into the many body effects responsible for these nonlinear signatures, we now use the moment analysis technique. This method is particularly well suited to quantitatively determine the characteristics of a band of arbitrary shape ${ }^{26}$ and is insensitive to most of the limitations mentioned above. The oscillator strength $f_{0}^{i i}$, the mean energy $E_{0}^{i i}$ and the width $\Gamma_{0}^{i i}$ are defined respectively as the 0th, 1st and 2nd moment of the linear absorption of the corresponding line. This method does not require any assumption regarding the line-shape of the resonances, all the parameters being defined in an intrinsic way through integrals of the spectra near the resonances. Practically, the oscillator strength reads $f_{0}^{i i}=\int_{\Delta E} \alpha^{i i}(E) \mathrm{d} E /(\Delta E)$, the energy position of the resonance reads : $E_{0}^{i i}=$ $\int_{\Delta E} E \alpha^{i i}(E) \mathrm{d} E /\left(f_{0}^{i i} \Delta E\right)$ and the width $\Gamma_{0}^{i i}$ is given by $:\left(\Gamma_{0}^{i i}\right)^{2}=\int_{\Delta E}\left(E-E_{0}^{i i}\right)^{2} \alpha^{i i}(E) \mathrm{d} E /\left(f_{0}^{i i} \Delta E\right)$. Applied to the non-linear spectra, this method gives access to the elementary deformations as a function of the time elapsed since excitation (Fig. 3 (a) and (b)) according to :

$$
\Delta f^{i i}(t)=\frac{1}{\Delta E} \int_{\Delta E} \Delta \alpha^{i i}(E, t) \mathrm{d} E
$$

for the oscillator strength,

$$
\Delta E^{i i}(t)=E^{i i}(t)-E_{0}^{i i}
$$

with

$$
E^{i i}(t)=\frac{1}{f_{0}^{i i} \Delta E} \int_{\Delta E} E\left[\alpha_{0}^{i i}(E)+\Delta \alpha^{i i}(E, t)\right] \mathrm{d} E
$$

for the energy shift and

$$
\Delta \Gamma^{i i}(t)=\Gamma^{i i}(t)-\Gamma_{0}^{i i}
$$

with

$$
\left(\Gamma^{i i}(t)\right)^{2}=\frac{1}{f_{0}^{i i} \Delta E} \int_{\Delta E}\left[E-E^{i i}(t)\right]^{2} \cdot\left[\alpha_{0}(E)+\Delta \alpha^{i i}(E, t)\right] \mathrm{d} E
$$

for the broadening. We used an integration window of $[1.16 \mathrm{eV}-1.36 \mathrm{eV}]$ and $[1.95 \mathrm{eV}-2.55 \mathrm{eV}]$ for analyzing the nonlinear changes of the $S_{11}$ and $S_{22}$ transitions respectively. Basically, Fig. 3 shows that the three contributions to the $S_{11}$ nonlinear spectra are of similar amplitude and thus are of equal importance to describe appropriately the nonlinear signals.

We first focus on the reduction of oscillator strength in each subband. Indeed, we shall see in the theoretical section (Sec. IV) that it fairly reflects the excitonic population of the same subband $\left(\Delta f^{i i} \propto n^{i i}\right)$. In order to follow the excitonic population relaxation in the first and second subband, we thus compare the dynamics of their respective $\Delta f$ after excitation on $S_{11}$ (Fig. 3 (a) 


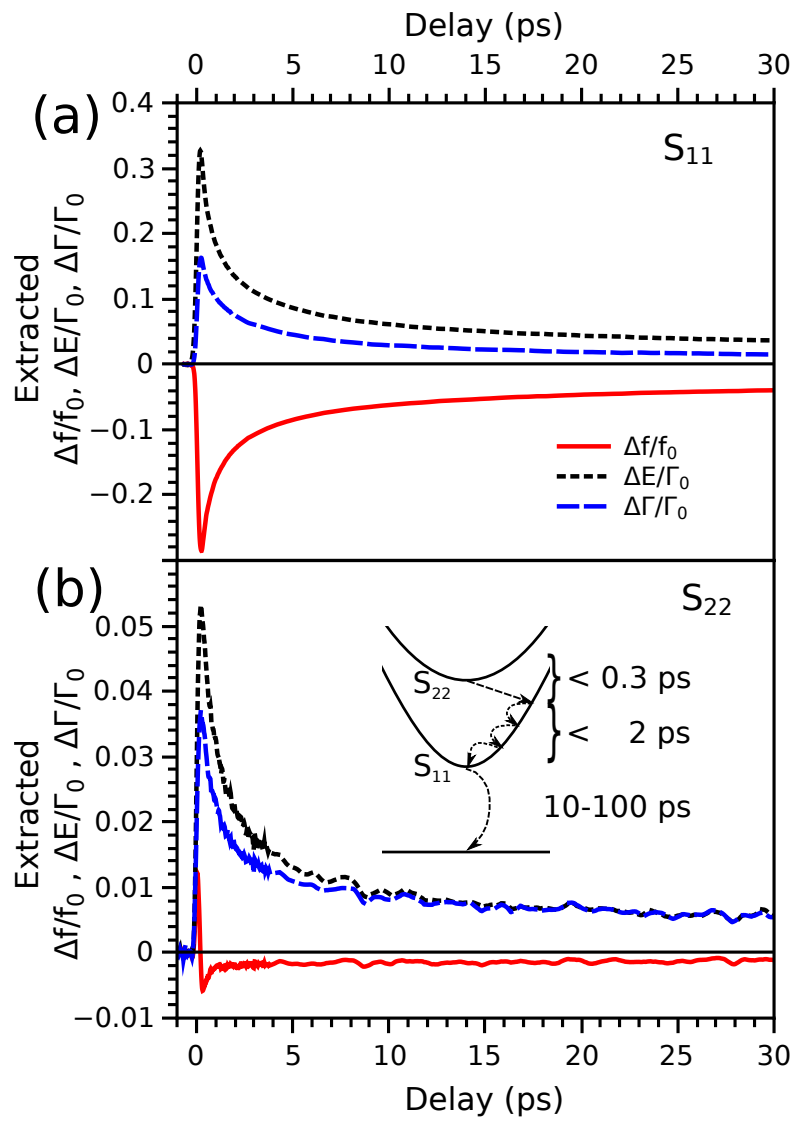

FIG. 3. (color online) Relaxation dynamics of the elementary deformations of $S_{11}$ (a) and $S_{22}$ (b) bands with $S_{11}$ excitation at a fluence of $3.2 \times 10^{14}$ photons.cm ${ }^{-2}$.

and (b)). Strikingly, $\Delta f^{22} / f_{0}^{22}$ becomes completely negligible within a few ps, whereas $\Delta f^{11} / f_{0}^{11}$ remains sizable for several tens of ps. This means that the $S_{22}$ population becomes negligible within a ps even though a small up-conversion from the $S_{11}$ state can be seen in the very early delays. Note that the same ultra-fast disappearance of the $S_{22}$ population is observed upon direct pumping of the $S_{22}$ transition or at higher energy (not shown), which confirms that the dynamics of the two types of excitons is very different, the upper one showing a much reduced lifetime ${ }^{27,28}$. We emphasize that the dynamics of $\Delta f^{22}$ is quite singular as compared to the dynamics of the change of absorption of this band. This means that the bleaching of the $S_{22}$ band originates from other processes than band filling. We end up with the picture that within a few ps electron-hole pairs populating $S_{11}$ states are predominant in the nanotube. The absence of $S_{22}$ population after excitation by the pump pulse is consistent with calculations of exciton relaxation from $S_{22}$ to $S_{11}$ implying efficient TO and LA-phonon emission in less than $300 \mathrm{fs}^{29}$ (Inset of Fig. 3).

$\Delta E^{11}$ and $\Delta \Gamma^{11}$ are plotted against $\Delta f^{11}$ in Fig. 4. The experimental values of the broadening are compatible with our previous studies using spectral hole-burning experiments $^{9}$ although the experimental conditions are quite different (chiral distribution, temperature...) showing that these nonlinear properties are essentially intrinsic. As we shall see in Sec. IV, the energy shift and broadening naturally scale with the exciton binding energy $E_{b}$. Our study shows that $\Delta \Gamma^{11} / E_{b}=0.05 \Delta f^{11} / f_{0}$ with $E_{b}=350 \mathrm{eV}$, which is consistent with the relation $\Delta \Gamma^{11} / E_{b}=[0.02-0.1] \Delta f^{11} / f_{0}$ obtained by Nguyen et $a l .{ }^{9}$ We note that this previous study did not mention any spectral shift. This actually points an important difference between the two methods. Spectral hole burning experiments in an inhomogeneous distribution yield an average of the nonlinear signals over a spectral window equivalent to the homogeneous line-width. In contrast to the oscillator strength reduction and to the broadening, the change of absorption due to an energy shift is an odd function with a vanishing average value. We actually estimated that in our previous (hole burning) study the residual effect of a spectral shift would be at least one order of magnitude lower than the two other contributions ${ }^{9}$ due to this averaging effect.

In contrast, in this chiral enriched sample showing little inhomogeneous broadening, we observe that the photoexcitation of carbon nanotubes consistently leads to a blue shift of the excitonic resonances. We note that such a blue-shift of a few meV at high excitation is commonly observed in other semiconductor nanostructures (see for instance references $\left.{ }^{26,30}\right)$. In the case of carbon nanotubes a blue shifted PA was reported in several studies for short time-delays, whereas at longer time delays, transient spectra were tentatively interpreted with red shifts of the lines ${ }^{8,10,13,31}$. We do not observe such red shift in our data. This inconsistency in the literature is probably due to the use of poly-disperse samples in several of these studies that prevented a fine identification of the spectral changes. As a consequence, the physical interpretation also remained quite elusive : these PA bands were tentatively assigned to transitions to the bi-exciton ${ }^{10}$, to a speculative transition to the two-exciton $S_{11}$ manyfold $^{13}$, to the Stark shift induced by photo-induced free carriers $^{31}$ or to the coupling with non-equilibrium hot phonons ${ }^{8}$.

Finally, we observe a linear relationship between all the nonlinear quantities over time, which clearly shows that they share the same dynamics, independently of the pump power, in agreement with a common physical origin. Quantitatively, this linear relationship reads $\Delta E^{11}(\mathrm{meV})=-(39 \pm 5) \Delta f^{11} / f_{0}^{11}$. The broadening of the $S_{11}$ resonance also presents a linear relationship with $\Delta f^{11}$ (inset of Fig. 4) : $\Delta \Gamma^{11}(\mathrm{meV})=-(19 \pm$ 5) $\Delta f^{11} / f_{0}^{11}$. Interestingly, Fig. 4 shows that despite a possibly complicated (multi-component) time decay, the evolution of one of these parameters as a function of another shows a simple linear relationship over a large span (two orders of magnitude) of initial carrier density. This shows that the three parameters share the same exciton density dependence (cf. Sec. IVD). 


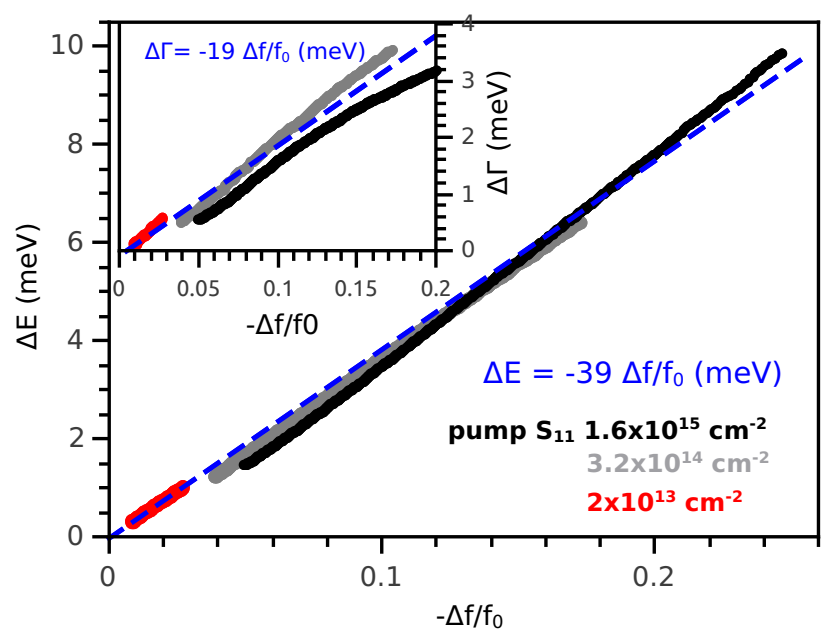

FIG. 4. Energy shift and broadening (inset) of the $S_{11}$ excitonic transition as a function of the change of oscillator strength of the same transition, extracted from the moment analysis (see text) for a resonant pumping of the $S_{11}$ transition and for several excitation densities.

\section{MODELING OF MANY-BODY EFFECTS}

In order to connect the macroscopic nonlinear signatures $\Delta f, \Delta E$ and $\Delta \Gamma$ to the underlying microscopic many-body effects, we developed a lowest order calculation of these effects by generalizing to the one-dimensional case the models established for twodimensional nanostructures ${ }^{14,15}$, that are based on a first order perturbative mean field approach. From a qualitative point of view, these many-body effects can be split into two main contributions. The first one is the so-called phase space filling (PSF) effect that is reminiscent of the Pauli principle acting on the fermionic components of the excitons (composite bosons). It mainly gives rise to a reduction of the oscillator strength $\Delta f_{P S F}$. The second one is related to the Coulomb interactions which give rise to several contributions, including short range effects like exchange interaction, direct interaction or exciton wavefunction renormalization (EWR) and long range effects (screening).

The contribution of the direct Coulomb interaction vanishes in the particular case of carbon nanotubes because of the identical effective masses of conduction electrons and holes ${ }^{32}$. For 2D nanostructures, the exchange of one fermion between two excitons gives rise to a blue shift as long as the momentum exchanged during the process is small as compared to the inverse of the excitonic Bohr radius ${ }^{15,33}$. In our experimental conditions, we therefore anticipate a blue shift due to this effect. The EWR results from the corrections to the exciton wave function when taking into account the Coulomb perturbation. It mainly redistributes the oscillator strength of the transition, giving an additional contribution $\Delta f_{E W R}$.
Finally, the screening of the Coulomb interaction by the population of excitons gives corrections to all the previously mentioned terms, leading to an overall redshift. The screening by excitons is however much weaker than that due to free carriers, especially in the case of carbon nanotubes where the small Bohr radius of the exciton reduces considerably their polarizability ${ }^{2}$. Furthermore, it is well established that such screening is strongly suppressed in reduced dimensionality systems ${ }^{14}$. In a classical picture, this results from the impossibility for counter charges to surround the test charge. In the following, the effects of excitonic screening will be neglected. As a consequence, the calculated nonlinear effects shall be considered as upper bounds. Finally, we also take into account the broadening resulting from the interaction between the excitons belonging either to the same or to separate subbands. This broadening arises from both the direct exciton-exciton annihilation processes (EEA) that give rise to a lifetime reduction and from quasi-elastic exciton-exciton scattering (EES) within a given subband or across a pair of bands (Fig. 5).

In line with previous works, we use the contact interaction approximation to regularize the Coulomb interaction (Eq. 1), which is justified by the very small Bohr radius in carbon nanotubes ${ }^{24}$.

$$
V_{\text {contact }}=-U \delta(r)
$$

with $U>0$. The value of $U$ is set by the scaling law with the exciton binding energy $E_{b}$, which is known from experiments ${ }^{2,34}$.

$$
E_{b}=\frac{\mu U^{2}}{2 \hbar^{2}}=\frac{\hbar^{2}}{2 \mu r_{B}^{2}}
$$

where $\mu$ stands for the reduced electron-hole effective mass and $r_{B}$ stands for the exciton Bohr radius.

Finally, the $S_{11}$ exciton relative motion wave-function for the $1 S$ state reads :

$$
\varphi(r)=\frac{1}{\sqrt{r_{B}}} \exp \left(-|r| / r_{B}\right)
$$

which gives in the $k$-space :

$$
\phi_{k}=2 \sqrt{\frac{r_{B}}{L}} \frac{1}{1+\left(r_{B} k\right)^{2}}
$$

where $L$ stands for the length of the nanotube so that $\sum_{k}\left|\phi_{k}\right|^{2}=1$.

\section{A. Reduction of oscillator strength}

The PSF contribution to the reduction of oscillator strength for a total number of excitons $N$ is obtained following Ref. ${ }^{14}$ : 


$$
f_{0} \propto \sum_{k, k^{\prime}} \phi_{k}^{*} \phi_{k^{\prime}} \quad \rightarrow \quad f \propto \sum_{k, k^{\prime}} \phi_{k}^{*}\left(1-N\left|\phi_{k^{\prime}}\right|^{2}\right) \phi_{k^{\prime}}
$$

which yields up to first order in the exciton density $n$ :

$$
\left.\frac{\Delta f}{f_{0}}\right|_{P S F}=-N \frac{\sum \phi_{k}^{3}}{\sum \phi_{k}}=-\frac{3}{2} r_{B} n
$$

Beyond phase space filling, an important consequence of the presence of excitons arises from the Coulomb interaction. To first order in perturbation theory, the Coulomb term gives a modification of the exciton wave function, which in turn leads to an additional contribution to the change of oscillator strength of the excitonic transition. We obtain :

$$
\left.\frac{\Delta f}{f_{0}}\right|_{E W R}=\frac{1}{\sum \phi_{k}} \sum_{k^{\prime}} \frac{\left\langle 1 S\left|H-H_{0}\right| k^{\prime}\right\rangle}{-\left(E_{b}+\frac{\hbar^{2} k^{\prime 2}}{2 \mu}\right)}+c . c .
$$

where $|1 S\rangle=\sum_{k} \phi_{k}|k\rangle$ and c.c. stands for the complex conjugate. The calculation yields :

$$
\begin{aligned}
\left.\frac{\Delta f}{f_{0}}\right|_{E W R} & =-\frac{N}{E_{b} \sum \phi_{k}} \sum_{k, k^{\prime}} \frac{V_{k, k^{\prime}}\left(\phi_{k}^{3}-\phi_{k}^{2} \phi_{k^{\prime}}\right)}{1+\frac{\hbar^{2} k^{\prime 2}}{2 \mu E_{b}}}+c . c .(8) \\
& =-r_{B} n
\end{aligned}
$$

Finally, the total reduction of oscillator strength reads :

$$
\left.\frac{\Delta f}{f_{0}}\right|_{\text {tot }}=\left.\frac{\Delta f}{f}\right|_{P S F}+\left.\frac{\Delta f}{f}\right|_{E W R}=-\frac{5}{2} r_{B} n
$$

\section{B. Energy shift}

To first order in perturbation theory, this Coulomb interaction can be treated in the Hartree-Fock approximation and leads to an energy shift of the excitonic transition that can be calculated as follows:

$$
\begin{aligned}
\Delta E=E-E_{0} & =\left\langle 1 S\left|H-H_{0}\right| 1 S\right\rangle \\
& =\sum_{k, k^{\prime}} \phi_{k}^{*}\left\langle k\left|H-H_{0}\right| k^{\prime}\right\rangle \phi_{k^{\prime}}
\end{aligned}
$$

where

$$
\left\langle k\left|H-H_{0}\right| k^{\prime}\right\rangle=-\delta_{k, k^{\prime}} \sum_{k^{\prime \prime}} V_{k k^{\prime \prime}} N\left|\phi_{k^{\prime \prime}}\right|^{2}+V_{k k^{\prime}} N\left|\phi_{k}\right|^{2}
$$

Using the contact interaction approximation (Eq. 1), yields :

$$
\Delta E=\frac{U}{2} n=E_{b} r_{B} n
$$

\section{Broadening}

Exciton-exciton scattering results in a global broadening of the lines. This exciton collisional broadening consists of two contributions, namely the exciton-exciton annihilation (EEA) and the quasi-elastic exciton-exciton scattering $(\mathrm{EES})^{9}$. In the former process, the collision between two $S_{11}$ excitons results in the annihilation of one of them whereas the second one is promoted in a higher level. This process is responsible for the exciton population decay observed in time-resolved measurements (Sec. III). This reduced population lifetime automatically results in a minimum broadening of the line given by $(\hbar / n)(\mathrm{d} n / \mathrm{d} t)$. Using the proportionality between the reduction of oscillator strength $\Delta f / f_{0}$ and the exciton population (Eq. 10), we estimated this contribution by computing numerically the quantity $\left(f_{0} / \Delta f\right)\left(\mathrm{d}\left(\Delta f / f_{0}\right) / \mathrm{d} t\right)$ from the data of Fig. 3. We found that the broadening induced by the diffusion-limited Auger scattering reaches a maximum of $0.2 \mathrm{meV}$ in the first picosecond and remains below $0.02 \mathrm{meV}$ for delays larger than $5 \mathrm{ps}$. This EEA induced broadening is much smaller than the experimentally observed broadening (Figs. 3, 4). Therefore, we conclude that EEA brings a negligible contribution to the global broadening.

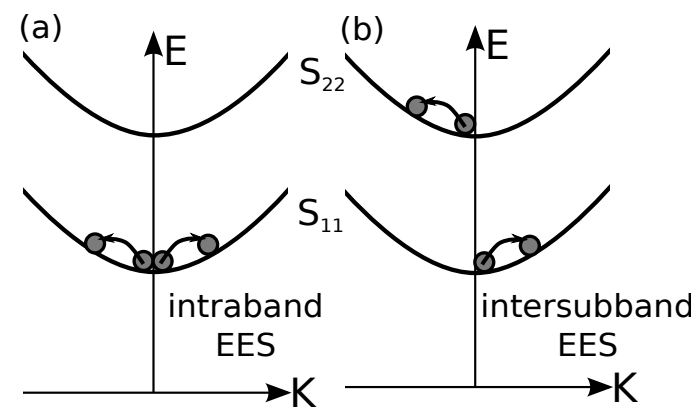

FIG. 5. Exciton energy dispersion and schematic representation of the exciton-exciton scattering process for the intraband case (a) and intersubband case (b).

In the EES process the dephasing of the $S_{11}$ transition results from quasi-elastic intraband exciton-exciton scattering ${ }^{15}$ (Fig. 5(a)). This scattering rate can be calculated in a self-consistent way until it remains small with respect to the natural line-width of the transition. This is always the case in our experimental conditions where $\Gamma_{0} \simeq 25 \mathrm{meV}$ while $\Delta \Gamma \leq 5 \mathrm{meV}$. Following Ref. ${ }^{9}$, we obtain :

$$
\Delta \Gamma_{E E S}=2 E_{b} r_{B} n \xi(b)
$$

Where $\xi(b)=\frac{4}{\pi} b \int \mathrm{d} q \frac{|I(q)|^{2}}{1+b^{2} q^{4}}$ and $|I(q)|=\frac{16}{\left(q^{2}+4\right)^{2}}-$ $\frac{6}{\left(q^{2}+4\right)\left(q^{2}+1\right)}$, with $b=\hbar^{2} /\left(8 \mu r_{B} \Gamma\right)$. For $E_{b}=350 \mathrm{meV}$, giving $r_{B}=1.5 \mathrm{~nm}$, and $\Gamma \simeq \Gamma_{0}=25 \mathrm{meV}$, we find $\xi(b)=0.71$. In total, the overall broadening reads : 


$$
\Delta \Gamma=1.4 E_{b} r_{B} n
$$

\section{Comparison with experiments}

We first note that our theoretical approach is based on a first order perturbative theory which leads to corrections that are linear in exciton density. These corrections may however be nonlinear in pump intensity since the photo-created population may result from nonlinear population decay mechanisms (such as Auger processes for instance). Since the evaluation of the exciton population is always indirect, we rather choose to work with ratios of nonlinear quantities (like energy shift and broadening for instance) in order to eliminate this exciton density. Importantly, the fact that we observe a linear behavior (see Fig. 4, 6) means that the first order approximation is sufficient. Quantitatively, we estimate that the exciton density we reach in the present experiments never exceeds $10 \%$ of the Mott density $1 / r_{B}$, which ensures that second order corrections are at least one order of magnitude weaker than the main contribution.

We first discuss the nonlinear shift of the excitonic resonances. Importantly, this correction mainly results from the exchange interaction and always gives a blue shift of the excitonic transition. This effect is usually suppressed in 3D materials due to the long range screening which is no longer efficient in reduced dimensionality. This prediction is in striking agreement with our experimental observations that consistently show a blue shift whatever the excitation density or wavelength.

The most convenient way to make a quantitative comparison between the theoretical predictions and the experimental results is to compare the energy shift to the relative reduction of oscillator strength (Fig. 4). By doing so, we can eliminate the exciton density and the saturation density $\left(1 / r_{B}\right)$, both of which are difficult to assess accurately. The only free parameter in this comparison is the exciton binding energy. This latter is known from previous experimental studies based on two-photon fluorescence spectroscopy ${ }^{2,34}$ and is of the order of $350 \mathrm{meV}$. Importantly, this value is highly sensitive to the microscopic dielectric surrounding of the nanotube, but the sample of the present study and that of Refs. ${ }^{2,34}$ are very similar to this respect. Finally, we obtain from our measurements the constant ratio $\frac{\Delta E^{11} / E_{b}}{\Delta f^{11} / f_{0}} \simeq-0.13 \pm 0.01$, whereas the theoretical prediction yields -0.4 . Thus, theory and experiment agree within a factor of 3 , which is satisfactory in terms of order of magnitude. Several reasons can explain the residual discrepancy. Obviously, screening (that was neglected in our calculation) would redshift the transition, which would reduce the predicted blue shift and bring it closer to our observations. However, as already discussed above we do not anticipate this effect to be strong for excitons in a confined geometry. Another possibility would be that some of the excitons split into free electron-hole pairs through exciton-exciton collision, leading to much stronger screening ${ }^{31,35,36}$. In the same time, it was predicted that such electron-hole plasma would enhance the reduction of oscillator strength when the temperature is much lower than the exciton binding energy, which is the case for carbon nanotubes ${ }^{14}$. Another limitation of the model above lies in the contact interaction approximation that may overestimate the short range interaction at the expense of the long ones, leading again to a global overestimate of the blue shift.

Regarding broadening, our measurements give the constant ratio $\frac{\Delta \Gamma / E_{b}}{\Delta f / f_{0}} \simeq-0.05 \pm 0.01$ (cf. Inset of Fig. 4), whereas the theoretical prediction yields -0.57 (Eqs. 10 and 16). Similarly to the blue shift effect, the absence of screening of interactions by electron-hole pairs may explain the excessive theoretical value for the broadening. In addition, we note that the contact interaction approximation yields an underestimate of the excitonic Bohr radius ${ }^{37}$, which in turn leads to an overestimate of the EES broadening. For instance, if we allow the Bohr radius to reach $2.5 \mathrm{~nm}$, in agreement with several experimental reports ${ }^{9,38}$, we obtain $\Delta \Gamma=0.45 E_{b} \cdot r_{B} \cdot n$ and thus $\frac{\Delta \Gamma / E_{b}}{\Delta f / f_{0}} \simeq 0.2$ in agreement with the experimental observations within a factor of 4 .

\section{INTERSUBBAND TRANSITIONS}

In agreement with previous reports ${ }^{8,13,31}$, we observe a nonlinear signal at the $S_{22}$ transition upon resonant excitation of the $S_{11}$ transition. Remarkably, we show that this signal consists in energy shift and broadening contributions only. The absence of oscillator strength reduction of the $S_{22}$ transition after 1 ps shows that there is no significant population created on $S_{22}$ upon $S_{11}$ excitation. As we shall see in the following sections, energy shift and collisional broadening may arise from intersubband processes. In contrast, such intersubband processes do not give rise to any significant change of oscillator strength. Getting back to the model of Sec. IV, we observe that the PSF contribution vanishes for such intersubband processes since the probed excitonic states $\left(S_{22}\right)$ are not built from the states populated by the $S_{11}$ excitons. In addition, the EWR contribution to the reduction of oscillator strength is also strongly reduced for intersubband processes due to the denominator proportional to the energy difference ${ }^{14}$. We thus expect this contribution to be 5 to 10 times smaller than in the intraband case, in agreement with the experimental results.

This absence of a sizable $S_{22}$ population does not conflict with the $1 / \sqrt{t}$ decay law that implies a bimolecular exciton-exciton annihilation and possibly upper bands population through Auger up-conversion, but rather shows that the coupling between the upper and the lower subbands through phonon emission is so fast that the resulting population on $S_{22}$ remains negligible. 
This is consistent with previous experimental studies ${ }^{27,28}$ showing a intersubband coupling rate on the order of $20 \mathrm{ps}^{-1}$.

\section{A. Energy shift}

The nonlinear signal observed on the $S_{22}$ transition results only from interactions between $S_{11}$ excitons photocreated by the pump and $S_{22}$ excitons photo-created by the probe. These interactions lead to energy renormalization and collisional broadening through the same manybody mechanisms that give rise to similar signatures on $S_{11}$. Indeed, Fig. 6 shows a striking proportionality between the shifts of the upper and the lower exciton, with a proportionality factor of 0.6. A very similar behavior is observed for the broadening (Fig. 6 Inset) with a proportionality factor of 0.9 , showing that the scattering probability of a $S_{22}$ exciton onto an $S_{11}$ exciton is almost equal to that of the scattering between two $S_{11}$ excitons. Note that although these intersubband energy shift and broadening values are almost as large as the intraband ones, there is no contradiction with the 10 times smaller signal observed at the $S_{22}$ resonance : this simply stems from the much larger width of this resonance, which weakens the change of absorption induced by these shift and broadening and from the absence of reduction of oscillator strength for intersubband processes.

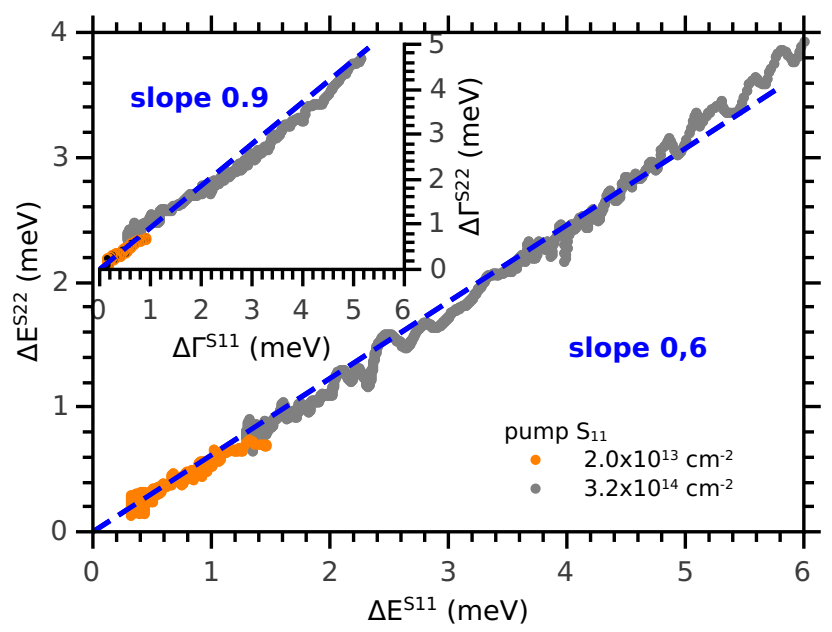

FIG. 6. Energy shift of the $S_{22}$ excitonic transition as a function of the one observed on the $S_{11}$ transition for a resonant pumping of the $S_{11}$ transition and for two excitation densities. Inset : broadening of the $S_{22}$ excitonic transition as a function of the one observed on the $S_{11}$ transition for a resonant pumping of the $S_{11}$ transition and for the same excitation densities.

We developed a simple extension of the model presented in the previous section to account for this effect. We recall that the $S_{22}$ exciton is predominantly built from states belonging to the second conduction and valence subbands of the nanotubes and are therefore distinct from those used to build the $S_{11}$ exciton. In order to compute the spectral shift, we generalize the approach developed by Parks et al. ${ }^{39}$ in the case of two-dimensional structures. We again consider a contact interaction potential and we restrict our calculations to the $1 s$ states of both the $S_{11}$ and the $S_{22}$ excitons. Their envelope wave-functions $\phi_{k}^{11}$ and $\phi_{k}^{22}$ are thus identical (Eq. 3) except for the value of the Bohr radius. Following, the calculations of Ref. ${ }^{39,40}$, we obtain :

$$
\Delta E^{22}=-\frac{1}{2}\left(R_{S_{22} S_{11} S_{11}}^{S_{22}}+R_{S_{11} S_{22} S_{11}}^{S_{22}}\right) \cdot n_{11}
$$

with :

$$
R_{\gamma_{1} \gamma_{2} \gamma_{3}}^{\mu}=\sum_{k, k^{\prime}} V_{k k^{\prime}}\left[\phi_{k^{\prime}}^{\mu} \phi_{k^{\prime}}^{\gamma_{1}} \phi_{k}^{\gamma_{2}} \phi_{k}^{\gamma_{3}}-\phi_{k^{\prime}}^{\mu} \phi_{k}^{\gamma_{1}} \phi_{k^{\prime}}^{\gamma_{2}} \phi_{k^{\prime}}^{\gamma_{3}}\right]
$$

This expression can be developed in the case of a contact interaction potential neglecting the dielectric screening effects ${ }^{40}$. We obtain :

$$
\begin{array}{r}
\Delta E^{22}=n_{11} \frac{U}{2} \sum_{k, k^{\prime}}\left[-\left(\phi_{k^{\prime}}^{22}\right)^{2}\left(\phi_{k}^{11}\right)^{2}+\phi_{k}^{22} \phi_{k^{\prime}}^{22}\left(\phi_{k^{\prime}}^{11}\right)^{2}-\right. \\
\left.\phi_{k}^{11} \phi_{k}^{22} \phi_{k^{\prime}}^{11} \phi_{k^{\prime}}^{22}+\phi_{k}^{11} \phi_{k^{\prime}}^{11}\left(\phi_{k^{\prime}}^{22}\right)^{2}\right]
\end{array}
$$

Remarkably, the sum equals 1 , leading to the simple result :

$$
\Delta E^{22}=n_{11} \frac{U}{2}=\Delta E^{11}
$$

This remarkably simple result shows that within the contact interaction approximation, the energy shifts of the $S_{22}$ and $S_{11}$ bands induced by the presence of $S_{11}$ excitons are identical. This is in good qualitative agreement with our experimental observation of a linear relationship between the shifts with a proportionality factor of 0.6 .

\section{B. Broadening}

One of the strong results of this study is the fact that the $S_{22}$ transition is broadened when a population of $S_{11}$ excitons is created. Quantitatively, this crossed broadening is almost equal to the direct one, that is the one observed on the $S_{11}$ transition itself (see inset of Fig. 6). This crossed broadening is reminiscent of the crossed energy shift described in the previous paragraph. Actually, as in the intraband case, the intersubband broadening can be understood within the framework of collisional broadening (Fig. 5(b) $)^{15}$. Qualitatively, the wave-functions of the excitons belonging to different subbands differ only by the extension of the bound 
relative motion along the tube axis and by a subbanddependent circumferential phase (related to the pseudo angular momentum $)^{41}$. However, the matrix element of the Coulomb interaction is insensitive to the phase factors (the angular momentum is conserved separately for each exciton in either intraband and crossed scatterings since in both cases pictured in Fig. 5 the carriers do not change subband). In addition, since the exciton binding energies are very similar for the $S_{11}$ and $S_{22}$ bands, the envelope of the exciton wave-functions have a similar spatial extension, leading to quasi-unity wave-function overlap $^{37}$. In total, although the numerical outcome of the matrix elements of the Coulomb interaction may differ slightly in the intraband and intersubband cases, they both rely on the same selection rules, leading to similar overall collision probabilities in agreement with the experimental observations.

\section{CONCLUSION}

In this study, we have conducted a thorough analysis of the nonlinear absorption spectrum of a $(6,5)$ enriched suspension of carbon nanotubes by using a broadband detection scheme covering the first and second excitonic bands. By using intrinsic analysis methods (moment analysis) that do not require any assumption about the line-shape, we were able to describe quantitatively the nonlinear spectra with only three elementary deformations of the linear spectrum, namely a reduction of oscil- lator strength, an energy shift and a broadening of the lines. In turn, these generic quantities were connected to a microscopic many-body model involving phase space filling and Coulomb interactions. Importantly, we have shown that the best way to compare this model to the experimental data, is to handle ratios of two of the elementary deformations in order to eliminate the influence of the exciton density, which is always difficult to assess experimentally. In particular, these results clarify the origin of the bleaching of the second exciton $\left(S_{22}\right)$ upon pumping the first one. We demonstrate that this bleaching originates from energy shift and broadening contributions only, ruling out the creation of a significant population in the $S_{22}$ level. In fact, we show that intersubband Coulomb interactions play a key role in the nonlinear properties of carbon nanotubes, showing up as collisional broadening between excitons belonging to different subbands or energy shifts subsequent to the presence of excitons in lower subbands. Importantly, we have shown both experimentally and theoretically that the magnitude of these intersubband processes is comparable to the intraband ones, leading to strong intersubband coupling in the nonlinear spectra of carbon nanotubes.

\section{ACKNOWLEDGEMENT}

The authors are thankful to Ermin Malic and Robson Ferreira for fruitful discussions. CV and GC are members of "Institut Universitaire de France".
* christophe.voisin@lpa.ens.fr

1 S. Reich, C. Thomsen, and J. Maultzsch, Carbon nanotubes : basic concepts and physical properties (WileyVCH, Weinheim; Cambridge, 2004).

2 F. Wang, G. Dukovic, L. E. Brus, and T. F. Heinz, Science 308, 838 (2005).

3 C. Voisin, S. Berger, S. Berciaud, H. Yan, J.-S. Lauret, G. Cassabois, P. Roussignol, J. Hone, and T. F. Heinz, Phys. Stat. Sol. (b) 245, 900 (2012).

${ }^{4}$ F. Wang, G. Dukovic, E. Knoesel, L. E. Brus, and T. F. Heinz, Phys. Rev. B 70, 241403 (2004).

5 L. Colombier, J. Selles, E. Rousseau, J. S. Lauret, F. Vialla, C. Voisin, and G. Cassabois, Phys. Rev. Lett. 109, 197402 (2012).

${ }^{6}$ S. M. Santos, B. Yuma, S. Berciaud, J. Shaver, M. Gallart, P. Gilliot, L. Cognet, and B. Lounis, Phys. Rev. Lett. 107, 187401 (2011).

7 A. Martinez and Z. Sun, Nat. Photon. 7, 842 (2013).

8 T. Koyama, S. Yoshimitsu, Y. Miyata, H. Shinohara, H. Kishida, and A. Nakamura, J. Phys. Chem. C 117, 20289 (2013).

9 D. T. Nguyen, C. Voisin, P. Roussignol, C. Roquelet, J. S. Lauret, and G. Cassabois, Phys. Rev. Lett. 107, 127401 (2011).

10 D. J. Styers-Barnett, S. P. Ellison, B. P. Mehl, B. C. Westlake, R. L. House, C. Park, K. E. Wise, and J. M. Pa- panikolas, J. Phys. Chem. C 112, 4507 (2008).

11 Y.-Z. Ma, C. D. Spataru, L. Valkunas, S. G. Louie, and G. R. Fleming, Phys. Rev. B 74, 085402 (2006).

12 H. Zhao, S. Mazumdar, C.-X. Sheng, M. Tong, and Z. V. Vardeny, Physical Review B 73, 075403 (2006).

13 Z. Zhu, J. Crochet, M. S. Arnold, M. C. Hersam, H. Ulbricht, D. Resasco, and T. Hertel, J. Phys. Chem. C 111, 3831 (2007).

14 S. Schmitt-Rink, D. S. Chemla, and D. A. B. Miller, Phys. Rev. B 32, 6601 (1985).

15 C. Ciuti, V. Savona, C. Piermarocchi, A. Quattropani, and P. Schwendimann, Phys. Rev. B 58, 7926 (1998).

16 P. M. Vora, X. Tu, E. J. Mele, M. Zheng, and J. M. Kikkawa, Phys. Rev. B 81, 155123 (2010).

17 F. Vialla, E. Malic, B. Langlois, Y. Chassagneux, C. Diederichs, E. Deleporte, P. Roussignol, J.-S. Lauret, and C. Voisin, Phys. Rev. B 90, 155401 (2014).

18 A. V. Naumov, S. Ghosh, D. A. Tsyboulski, S. M. Bachilo, and R. B. Weisman, ACS Nano 5, 1639 (2011).

19 S. A. Kovalenko, A. L. Dobryakov, J. Ruthmann, and N. P. Ernsting, Phys. Rev. A 59, 2369 (1999).

20 D. Stich, F. Späth, H. Kraus, A. Sperlich, V. Dyakonov, and T. Hertel, Nature Photonics 8, 139 (2013).

21 J. Allam, M. T. Sajjad, R. Sutton, K. Litvinenko, Z. Wang, S. Siddique, Q.-H. Yang, W. H. Loh, and T. Brown, Phys. Rev. Lett. 111, 197401 (2013). 
${ }^{22}$ M. Hoyuelos and H. O. Mártin, Phys. Rev. E 48, 3309 (1993).

23 D. Ben-Avraham and S. Redner, Phys. Rev. A 34, 501 (1986).

24 F. Wang, Y. Wu, M. S. Hybertsen, and T. F. Heinz, Phys. Rev. B 73, 245424 (2006).

25 B. Yuma, S. Berciaud, J. Besbas, J. Shaver, S. Santos, S. Ghosh, R. B. Weisman, L. Cognet, M. Gallart, M. Ziegler, B. Hönerlage, B. Lounis, and P. Gilliot, Phys. Rev. B 87, 205412 (2013).

${ }^{26}$ K. Litvinenko, D. Birkedal, V. G. Lyssenko, and J. M. Hvam, Phys. Rev. B 59, 10255 (1999).

27 J. S. Lauret, C. Voisin, G. Cassabois, C. Delalande, P. Roussignol, O. Jost, and L. Capes, Phys. Rev. Lett. 90, 057404 (2003).

28 C. Manzoni, A. Gambetta, E. Menna, M. Meneghetti, G. Lanzani, and G. Cerullo, Phys. Rev. Lett. 94, 207401 (2005).

29 O. A. Dyatlova, C. Koehler, P. Vogel, E. Malic, R. M. Jain, K. C. Tvrdy, M. S. Strano, A. Knorr, and U. Woggon, Phys. Rev. B 90, 155402 (2014).

30 D. Hulin, A. Mysyrowicz, A. Antonetti, A. Migus, W. T. Masselink, H. Morkoc, H. M. Gibbs, and N. Peyghambarian, Phys. Rev. B 33, 4389 (1986).
31 G. Soavi, F. Scotognella, D. Brida, T. Hefner, F. Spth, M. R. Antognazza, T. Hertel, G. Lanzani, and G. Cerullo, J. Phys. Chem. C 117, 10849 (2013).

32 G. D. Mahan, ed., Many-particle physics. (Springer, 2000).

${ }^{33}$ G. Manzke, K. Henneberger, and V. May, Phys. Stat. Sol. (b) 139, 233 (1987).

34 J. Maultzsch, R. Pomraenke, S. Reich, E. Chang, D. Prezzi, A. Ruini, E. Molinari, M. S. Strano, C. Thomsen, and C. Lienau, Phys. Rev. B 72, 241402 (2005).

35 Y. Kumamoto, M. Yoshida, A. Ishii, A. Yokoyama, T. Shimada, and Y. K. Kato, Phys. Rev. Lett. 112, 117401 (2014).

36 W. H. Knox, R. L. Fork, M. C. Downer, D. A. B. Miller, D. S. Chemla, C. V. Shank, A. C. Gossard, and W. Wiegmann, Phys. Rev. Lett. 54, 1306 (1985).

37 V. Perebeinos, J. Tersoff, and P. Avouris, Phys. Rev. Lett. 92, 257402 (2004).

38 L. Lüer, S. Hoseinkhani, D. Polli, J. Crochet, T. Hertel, and G. Lanzani, Nat. Phys. 5, 54 (2008).

39 A. M. Parks, M. M. Dignam, and D. Wang, Phys. Rev. B 87, 205306 (2013).

40 A. M. Parks, Excitonic analysis of many body effects on the $1 s-2 p$ intraband transition in semi-conductor systems., Ph.D. thesis, Queen's University (2011).

41 B. O. Tayo and S. V. Rotkin, Phys. Rev. B 86, 125431 (2012). 